\title{
Turbulence transmission in parallel relativistic shocks using ray tracing
}

\author{
J. Tammi ${ }^{1,2}$ and R. Vainio ${ }^{3}$ \\ 1 Tuorla Observatory, Väisälä Institute for Space Physics and Astronomy, Väisäläntie 20, 21500 Piikkiö, Finland \\ e-mail: joni.tammi@iki.fi \\ 2 UCD School of Mathematical Sciences, University College Dublin, Belfield, Dublin 4, Ireland \\ 3 Department of Physical Sciences, PO Box 64, 00014 University of Helsinki, Finland
}

Received 10 January 2006 / Accepted 6 September 2006

ABSTRACT

\begin{abstract}
We apply a semi-classical approach of handling waves as quasiparticle gas in a slowly varying flow - analogous to ray tracing - to calculate the Alfvén wave transmission parameters, the resulting cross-helicity of the waves and the scattering-centre compression ratio, for cases where the shock thickness is large enough for the turbulent waves in the plasma to see the transition of the background flow parameters as smooth and slowly varying. For nonrelativistic shocks the wave transmission produces similar effects on the downstream turbulence and the scattering-centre compression ratio as does the transmission through a step shock: the downstream Alfvén waves propagate predominantly towards the shock in the local plasma frame and, thus, the scattering-centre compression ratio is larger than the gas compression ratio. For thick relativistic shocks, however, we find qualitative differences with respect to the step-shock case: for low-Alfvénic-Mach-number shocks the downstream waves propagate predominantly away from the shock, and the scattering-centre compression ratio is lower than that of the gas. Thus, when taken into account, the Alfvén wave transmission can decrease the efficiency of the first-order Fermi acceleration in a thick relativistic shock.
\end{abstract}

Key words. turbulence - shock waves - waves

\section{Introduction}

A common approximation in shock-related particle acceleration studies involving the first-order Fermi mechanism is to assume the scattering turbulence frozen-in to the plasma. This is very well justified in cases where the speed of the turbulent waves is negligibly small compared to the flow speed. For cases where the wave speed increases and becomes a notable fraction of the flow speed, however, the deviation of the scattering centre speed from the speed of the background flow can have notable effects on the particle acceleration scenario; it was noted already by Bell (1978) that the speed of the scattering centres is not necessarily that of the flow. Furthermore, the compression ratio (of the scattering centres) is known to have significant effect on the powerlaw spectral index of particles accelerated in the shock for both steplike and thick shocks (for modified shocks see Drury et al. 1982 and Drury 1983 for nonrelativistic analytical studies, and Virtanen \& Vainio 2005a, hereafter VV05 ${ }^{1}$, for numerical results for all speeds, and Keshet \& Waxman 2005 for an analytical approach for step shocks). The turbulent waves are, of course, also affected by the shock, and thus one has to start from the transmission of turbulence through the shock to arrive at describing particle acceleration in a case where wave-propagation effects are taken into account.

The effect of the transmission of Alfvén waves through a step shock was solved at the nonrelativistic limit by Vainio \& Schlickeiser (1998) and later generalised to include also relativistic shocks by Vainio et al. (2003, hereafter referred to as VVS03; 2005). They found that, regardless of the shock

${ }^{1}$ In Eq. (1) of VV05 there is a typo in the exponent in the denominator of the last term: $r^{2}$ should be $r^{3}$. speed, waves initially in equipartition were propagating predominantly antiparallel to the flow direction (i.e. backwards toward the shock in the downstream plasma frame) after being transmitted through a step shock with Alfvénic Mach number sufficiently low to yield non-negligible wave speeds compared to the speed of the plasma flow. They showed that this leads to an increased scattering-centre compression ratio, i.e., that the scattering-centre speed can undergo compression that is significantly higher than the compression of the flow itself. This effect thus causes the first-order Fermi acceleration to lead to accelerated particle energy spectral indices harder than $\sim 2.2$, the wellknown outcome of parallel step shocks at the ultrarelativistic limit in case of isotropic particle scattering (e.g., Kirk \& Duffy 1999; Keshet \& Waxman 2005). This transmission analysis is, however, valid only for shocks of small thickness and waves with wavelengths much longer than the width of the shock transition.

In this paper we continue studying the transmission of Alfvén waves through parallel shocks, but take an approach opposite to the step-shock approximation of VVS03. We calculate the transmission coefficients and their derivative parameters (the average cross-helicity of the waves, and the compression ratio of the scattering centres) for a continuous flow profile, approximating the transmission of waves through a shock with thickness sufficiently large for the waves to see the flow parameters varying slowly. The transmission coefficients for the nonrelativistic thick-shock case were calculated by VV05.

In general, a single shock wave is not either a step-shock nor a thick shock: as the turbulence in the upstream medium has a broad-band spectrum, some waves will see the shock as a thick while others see it as a thin structure. In addition, some waves have wavelengths matching the shock thickness. Thus, the 
general theory of wave propagation can not rely on either the thick or thin shock approximation. However, the general theory is mathematically very cumbersome and we have here chosen to limit our discussion to waves with wavelengths much shorter than the shock thickness. Combining these results with our earlier ones (VVS03) with the opposite assumption gives the first qualitative view on turbulence transmission through parallel shocks with finite thickness.

\section{Alfvén wave transmission}

\subsection{Theoretical background}

We adopt the idea of treating the waves as a quasiparticle gas. For monochromatic waves, the number density of the wave quanta, or the wave action density, is $N^{\prime}=E^{\prime} / \omega^{\prime}$, where $E^{\prime}$ and $\omega^{\prime}$ are the energy density and the angular frequency of the waves in the plasma frame. We will use the prime to denote the quantities measured in the plasma frame, while unprimed values are measured in the shock frame. Below, we will consider a broad-band spectrum of waves and, thus, the evolution of the quasi-particle distribution function in the phase space $(\boldsymbol{x}, \boldsymbol{k})$.

Our approach is equivalent to the use of a variational principle in deriving the equation for the turbulent MHD wave-action density, pioneered by Dewar (1970) and extended to the relativistic case by Achterberg (1983).

In addition to the angular frequency $\omega$, we will be needing the wavenumber $k$ and frequency $f=\frac{\omega}{2 \pi}$, as well as their values in the local plasma frame $\left(\omega^{\prime}, k^{\prime}\right.$ and $f^{\prime}=\frac{\omega^{\prime}}{2 \pi}$ respectively). Because the medium consists of a time-independent flow profile through the shock, the shock-frame frequencies are conserved during shock crossing and we can write the correspondence between the shock- and plasma-frame values as

$k=\Gamma\left(k^{\prime}+V \omega^{\prime}\right)= \pm \Gamma\left(1 \pm V V_{\mathrm{A}}\right) \omega^{\prime} / V_{\mathrm{A}}$

and

$f=\Gamma\left(\omega^{\prime}+V k^{\prime}\right) /(2 \pi)= \pm \Gamma\left(V \pm V_{\mathrm{A}}\right) f^{\prime} / V_{\mathrm{A}}$,

where the dispersion relation $\omega^{\prime}= \pm V_{\mathrm{A}} k^{\prime}$ is used. The Alfvén speed $V_{\mathrm{A}}$ is obtained from the proper Alfvén speed (in units of c) as

$u_{\mathrm{A}}=\Gamma_{\mathrm{A}} V_{\mathrm{A}}=B_{0}^{\prime} / \sqrt{4 \pi \mu^{\prime} n^{\prime}}$,

where $\mu^{\prime}=\left(\rho^{\prime}+P^{\prime}\right) / n^{\prime}, n^{\prime}, \rho^{\prime}$ and $P^{\prime}$ are the specific enthalpy, the number density, the total energy density and the gas pressure, measured in the local plasma frame, and $B_{0}^{\prime}$ is the magnitude of the static large scale background magnetic field. For easier comparison with the step-shock transmission of VVS03 we will present results using the proper speed $u=\Gamma V$, where $\Gamma=1 / \sqrt{1-V^{2}}$ is the Lorentz factor, and the speeds are measured in units of $c$. Using subscripts 1 and 2 for far upstream and downstream values, respectively, we can introduce the Alfvénic Mach number of the shock $M^{2}=u_{1}^{2} / u_{\mathrm{A}, 1}^{2}$.

We adopt the turbulence spectra in a power-law form

$I^{\prime \pm}\left(k^{\prime}, x\right)=I_{0}^{\prime \pm} \cdot\left(k_{0}^{\prime} / k^{\prime}\right)^{q}$ for $k^{\prime}>k_{0}^{\prime}$,

at wavelengths greater than $k_{0}^{\prime}$, for waves flowing parallel (also referred to as forward waves, denoted by “+”) and antiparallel (or backward, denoted by "-") to the flow direction.

\subsection{Equation for the wave flux}

Let us consider the propagation of an Alfvén wave in a timeindependent medium with spatial gradients only in the direction of the background flow, aligned with the background magnetic field and the $x$-axis. The Hamiltonian of the system is

$H(\boldsymbol{x}, \boldsymbol{p})=\hbar \Omega\left(x, p_{x} / \hbar\right)$,

where $\boldsymbol{x}$ and $\boldsymbol{p}=\hbar \boldsymbol{k}$ are the canonical coordinates and momenta, $\boldsymbol{k}$ is the wavevector, and

$\omega=\Omega\left(x, k_{x}\right)=V_{\mathrm{g}}(x) k_{x}$

gives the dispersion relation of the Alfvén wave. Here $V_{\mathrm{g}}=\left(V+s V_{\mathrm{A}}\right) /\left(1+s V V_{\mathrm{A}}\right)$ is the group speed of the wave and $s= \pm 1$ gives its propagation direction with respect to the background plasma flow. Hamilton's equations of motion for the wave packet, thus, read

$\dot{x}=\frac{\partial H}{\partial p_{x}}=\frac{\partial \Omega}{\partial k_{x}} ; \quad \dot{k}_{x}=\frac{\dot{p}_{x}}{\hbar}=-\frac{1}{\hbar} \frac{\partial H}{\partial x}=-\frac{\partial \Omega}{\partial x}$,

with all other equations of motion being trivial: $\dot{y}=\dot{z}=\dot{p}_{y}=$ $\dot{p}_{z}=0$ because the Hamiltonian has no dependence on these phase space coordinates. Thus, the continuity equation for the phase space density,

$f(\boldsymbol{x}, \boldsymbol{p})=\frac{\mathrm{d} N}{\mathrm{~d}^{3} x \mathrm{~d}^{3} p}=\frac{1}{\hbar^{3}} \frac{\mathrm{d} N}{\mathrm{~d}^{3} x \mathrm{~d}^{3} k} \equiv \frac{1}{\hbar^{3}} N(\boldsymbol{x}, \boldsymbol{k})$,

of the waves (or Liouville's equation, equivalently) yields

$\frac{\partial N}{\partial t}+\frac{\partial}{\partial \boldsymbol{x}} \cdot(\dot{\boldsymbol{x}} N)+\frac{\partial}{\partial \boldsymbol{k}} \cdot(\dot{\boldsymbol{k}} N)=0$

which, after the substitution of the equations of motion, reduces to

$\frac{\partial N}{\partial t}+\frac{\partial}{\partial x}\left(\frac{\partial \Omega}{\partial k_{x}} N\right)-\frac{\partial}{\partial k_{x}}\left(\frac{\partial \Omega}{\partial x} N\right)=0$.

We can integrate this equation over the perpendicular wave numbers $k_{y}$ and $k_{z}$ to obtain an equation for the number-density of waves over $k_{x}$,

$n_{k_{x}}\left(\boldsymbol{x}, k_{x}\right)=\frac{\mathrm{d} N}{\mathrm{~d}^{3} x \mathrm{~d} k_{x}}=\iint \mathrm{d} k_{y} \mathrm{~d} k_{z} N(\boldsymbol{x}, \boldsymbol{k})$,

as

$\frac{\partial n_{k_{x}}}{\partial t}+\frac{\partial}{\partial x}\left(\frac{\partial \Omega}{\partial k_{x}} n_{k_{x}}\right)-\frac{\partial}{\partial k_{x}}\left(\frac{\partial \Omega}{\partial x} n_{k_{x}}\right)=0$,

because $\Omega$ does not depend on $k_{y}$ or $k_{z}$. This equation holds regardless of the form of the distribution function with respect to the wavenumbers $k_{y}$ and $k_{z}$. These are, of course, constants of motion for the waves in our system.

From now on, we will assume that the wavevector is aligned with the $x$-axis (i.e., that $k_{y}=k_{z}=0$ ) and simplify the notation by writing $k_{x}=k$. Thus, using the explicit form of the dispersion relation, we obtain in steady state

$\frac{\partial}{\partial x}\left(V_{\mathrm{g}} n_{k}\right)-\frac{\partial}{\partial k}\left(\frac{\partial V_{\mathrm{g}}}{\partial x} k n_{k}\right)=0$.

Consider, next, the wave-action density over frequency, $n_{\omega}=\mathrm{d} N /\left(\mathrm{d}^{3} x \mathrm{~d} \omega\right)=n_{k} / V_{\mathrm{g}}$, and change to using the position $x$ 
and the wave frequency $\omega=V_{\mathrm{g}}(x) k$ as the independent variables instead of $x$ and $k$. Thus,

$$
\begin{aligned}
\left(\frac{\partial}{\partial x}\right)_{k} & \rightarrow\left(\frac{\partial}{\partial x}\right)_{\omega}+\frac{\mathrm{d} V_{\mathrm{g}}}{\mathrm{d} x} \frac{\omega}{V_{\mathrm{g}}}\left(\frac{\partial}{\partial \omega}\right)_{x} \\
\left(\frac{\partial}{\partial k}\right)_{x} & \rightarrow V_{\mathrm{g}}\left(\frac{\partial}{\partial \omega}\right)_{x}
\end{aligned}
$$

and we obtain

$$
\begin{aligned}
0= & {\left[\left(\frac{\partial}{\partial x}\right)_{\omega}+\frac{\mathrm{d} V_{\mathrm{g}}}{\mathrm{d} x} \frac{\omega}{V_{\mathrm{g}}}\left(\frac{\partial}{\partial \omega}\right)_{x}\right]\left(V_{\mathrm{g}} \cdot V_{\mathrm{g}} n_{\omega}\right) } \\
& \quad-V_{\mathrm{g}}\left(\frac{\partial}{\partial \omega}\right)_{x}\left(\frac{\mathrm{d} V_{\mathrm{g}}}{\mathrm{d} x} \omega n_{\omega}\right) \\
= & V_{\mathrm{g}}\left(\frac{\partial}{\partial x}\left(V_{\mathrm{g}} n_{\omega}\right)\right)_{\omega} .
\end{aligned}
$$

In this equation $\omega$ appears only as a parameter numbering the modes, which can be all treated as monochromatic waves using the constancy of their flux

$V_{\mathrm{g}} n_{\omega}=n_{k}\left(x, \omega / V_{\mathrm{g}}(x)\right)$

with respect to position at constant $\omega$. Thus,

$n_{k}(x, k)=n_{k}\left(-\infty, k_{1}\right)$,

where $k_{1}(k, x)=V_{\mathrm{g}}(x) k / V_{\mathrm{g}, 1}$ is the far-upstream wavenumber of the wave with wavenumber $k$ at $x$.

\subsection{Wave transmission coefficients}

Next we calculate the transmission coefficients for Alfvén waves with wavelengths much shorter than the shock thickness.

Transmission coefficients are needed for solving the turbulence conditions at a given location in the shock. In contrast to the step shock case (VVS03) where part of the waves are reflected at the shock and change their mode (from parallel to antiparallel, or vice versa), in the case of slowly changing medium all the waves are transmitted through the shock without reflection, and the turbulence at a given location can be obtained from equation

$I^{\prime \pm}\left(k^{\prime}, x\right)=T_{ \pm}^{\prime 2}(x) \cdot I_{1}^{\prime \pm}\left(k^{\prime}\right)$,

where $I_{1}^{\prime \pm}\left(k^{\prime}\right)=I^{\prime \pm}\left(k^{\prime}, x=-\infty\right)$ are the turbulence spectra far upstream for parallel (+) and antiparallel (-) waves, and $T_{ \pm}^{\prime 2}(x)$ are the corresponding transmission coefficients, now to be solved.

The co-moving intensity of the waves is related to the number-density of wave quanta by

$$
\begin{aligned}
I^{ \pm}\left(x, k^{\prime}\right) & =\frac{\hbar \omega^{\prime} n_{k^{\prime}}^{\prime \pm}}{4 \pi}=\frac{V_{\mathrm{A}} k^{\prime}}{V_{\mathrm{g}}^{ \pm} k} \frac{\hbar \omega n_{k}^{ \pm}}{4 \pi} \\
& =\frac{V_{\mathrm{A}}}{V_{\mathrm{g}}^{ \pm} \Gamma\left(1 \pm V V_{\mathrm{A}}\right)} \frac{\hbar \omega n_{k}^{ \pm}}{4 \pi}=\frac{V_{\mathrm{A}}}{\Gamma\left(V \pm V_{\mathrm{A}}\right)} \frac{\hbar \omega n_{k}^{ \pm}}{4 \pi},
\end{aligned}
$$

where we have made use of the invariance of $n_{k}^{ \pm}$under Lorentz transformations along the $x$ axis. (This holds because of the Lorentz invariance of $N(\boldsymbol{x}, \boldsymbol{k})$ and of the perpendicular wavenumber element $\mathrm{d} k_{y} \mathrm{~d} k_{z}=\mathrm{d} k_{y}^{\prime} \mathrm{d} k_{z}^{\prime}$.) Thus, we get

$\frac{\Gamma\left(V \pm V_{\mathrm{A}}\right)}{V_{\mathrm{A}}} I^{\prime \pm}\left(x, k^{\prime}\right)=\frac{\hbar \omega n_{k}^{ \pm}}{4 \pi}=\frac{\Gamma_{1}\left(V_{1} \pm V_{\mathrm{A} 1}\right)}{V_{\mathrm{A} 1}} I_{1}^{\prime \pm}\left(k_{1}^{\prime}\right)$, where $\Gamma_{1}\left(V_{1} \pm V_{\mathrm{A}, 1}\right) k_{1}^{\prime}=\omega=\Gamma\left(V \pm V_{\mathrm{A}}\right) k^{\prime}$. For power-law spectra, $I_{1}^{\prime \pm}\left(k^{\prime}\right) \propto k^{\prime-q}$, we finally obtain

$I^{\prime \pm}\left(x, k^{\prime}\right)=I_{1}^{\prime \pm}\left(k^{\prime}\right) \frac{V_{\mathrm{A}}}{V_{\mathrm{A}, 1}}\left[\frac{\Gamma_{1}\left(V_{1} \pm V_{\mathrm{A} 1}\right)}{\Gamma\left(V \pm V_{\mathrm{A}}\right)}\right]^{q+1}$.

At the nonrelativistic limit in the far downstream, this reduces to (VV05)

$I_{2}^{ \pm}\left(k^{\prime}\right)=I_{1}^{ \pm}\left(k^{\prime}\right) \cdot T_{ \pm}^{\prime 2}=I_{1}^{ \pm}\left(k^{\prime}\right) r^{q+1 / 2}\left[\frac{M \pm 1}{M \pm r^{1 / 2}}\right]^{q+1}$,

where $r=V_{1} / V_{2}$ is the gas compression ratio and $M$ reduces to its nonrelativistic form, $M=V_{1} / V_{\mathrm{A}, 1}$.

\section{Results and discussion}

From this point on, we deal only with plasma-frame quantities, so we omit the primes in variable symbols and assume them being measured in the plasma rest frame, unless otherwise mentioned. The local plasma speeds $V$ and the location are, of course, still in the shock frame.

For a general view we first look at the case of vanishing upstream cross-helicity. The ratio of forward-to-backward waves in the downstream is

$\frac{I^{+}}{I^{-}}=\left[\frac{\left(V_{1}+V_{\mathrm{A}, 1}\right)\left(V_{2}-V_{\mathrm{A}, 2}\right)}{\left(V_{1}-V_{\mathrm{A}, 1}\right)\left(V_{2}+V_{\mathrm{A}, 2}\right)}\right]^{q+1}$

which, in contrast to transmission in step shocks, can be also greater than one (i.e., the downstream cross-helicity is positive) if $V_{\mathrm{A}, 2}<V_{\mathrm{A}, 1} / r$. This, however, can occur only if the shock is relativistic (for nonrelativistic shock $V_{\mathrm{A}, 2}=V_{\mathrm{A}, 1} / \sqrt{r}$ and $I^{+} / I^{-} \leq 1$ always). One immediately sees that, as a consequence, while the effect of the transmission in the nonrelativistic regime is very similar to that of both non- and fully relativistic step shocks, for relativistic thick shocks qualitative differences arise.

In the following sections we study the cross-helicity of the transmitted waves in the downstream, and its development throughout the shock. We also calculate the scattering centre compression ratios in the downstream.

\subsection{Far downstream cross-helicity}

Here we calculate the normalised cross-helicity of the waves,

$H_{\mathrm{c}}(k, x)=\frac{I^{+}(k, x)-I^{-}(k, x)}{I^{+}(k, x)+I^{-}(k, x)}$,

in the far downstream $\left(H_{\mathrm{c}, 2}\right)$ from a given upstream state $\left(H_{\mathrm{c}, 1}\right)$ as a function of different Alfvénic Mach numbers.

For high- $M$ shocks, where the effect of wave speeds is negligible, the downstream cross-helicity approaches, of course, the cross helicity upstream as $M \rightarrow \infty$. As the Alfvénic Mach number decreases and approaches the critical Mach number $M_{\mathrm{c}}=\sqrt{r}$ (i.e., as the downstream Alfvén speed starts to approach the downstream flow speed), the cross-helicity for nonrelativistic shocks starts to decrease and approach -1 (as shown by VV05), as for step shocks of all speeds (VVS03).

For relativistic speeds and Mach number a few times the critical $M_{\mathrm{c}}$, however, nearly all of the waves are streaming parallel to the flow and $H_{\mathrm{c}, 2} \rightarrow+1$ as $u_{1} \rightarrow \infty$ almost regardless of the upstream cross-helicity. For the lowest $M \gtrsim M_{\mathrm{c}}$ the crosshelicity drops to -1 as the downstream Alfvén speed becomes equal to the flow speed; this happens because the transmission 


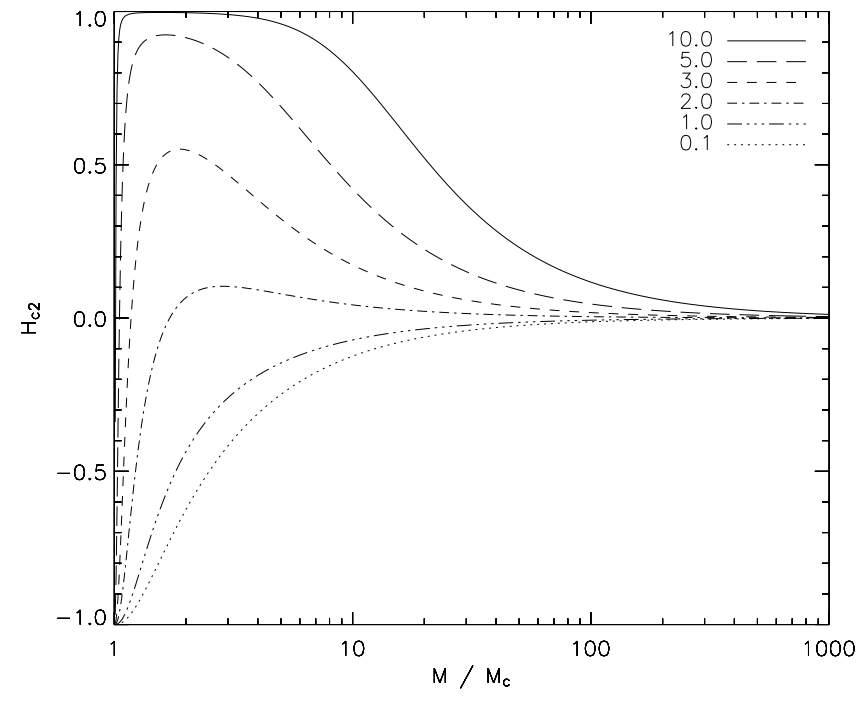

Fig. 1. The far downstream cross-helicity $H_{\mathrm{c}, 2}$ as a function of Alfvénic Mach number $M$ for different shock proper speeds $u_{1}=V_{1} \Gamma_{1} \cdot M_{\mathrm{c}}=\sqrt{r}$ is the critical Mach number, below which the downstream Alfvén speed exceeds the local plasma speed, and the shock becomes nonevolutionary. The spectral index of the turbulence power-spectrum is $q=1.5$

coefficients of the backward waves, $T_{-}^{2}$, goes to infinity. The physicality and removal of this mathematical singularity is discussed in Sect. 3.4.

The lack of wave reflection causes the special cases of only one upstream wave field to keep also the downstream wave field at the same state (i.e., $H_{\mathrm{c}, 1}= \pm 1 \Rightarrow H_{\mathrm{c}, 2}= \pm 1$ ) but the abovementioned general behaviour is seen for all $\left|H_{\mathrm{c}, 1}\right|<1$. The downstream cross-helicity as a function of the Alfvénic Mach number is plotted in Fig. 1 for non-to-highly relativistic shocks for vanishing upstream cross helicity.

\subsection{Cross-helicity as a function of location}

Next we want to study how the cross-helicity changes from the upstream value to that of the downstream. We use a hyperbolic tangent profile (Schneider \& Kirk 1989; Virtanen \& Vainio 2005) to describe the flow speed as a function of location, and follow the development of the local mean cross-helicity. Since the actual shock thickness is of no importance for the spatial properties of the test-wave transmission, as long as it is large enough to allow for the "smoothly-varying-background" assumption, we use only one flow profile (and one thickness) to demonstrate the general behaviour. The form of the flow speed profile is shown in the top panel of Fig. 2. The unit of location $x$ in that figure is arbitrary and is chosen so that the shock transition takes place within $\approx 1$ location unit.

While for nonrelativistic shocks the cross-helicity changes monotonically from the upstream value to that calculated in Sect. 3.1, for faster shocks the local cross-helicity $H_{\mathrm{c}}(x)$ has non-monotonic behaviour: the cross-helicity forms a maximum near the "centre" of the flow profile, where the velocity gradient has its largest value, after which it decreases to the downstream value $H_{\mathrm{c} 2}$.

An example of the non-monotonic cross-helicity is shown in Fig. 2, where the local cross-helicity $H_{\mathrm{c}}(x)$ (here set equal to zero the far upstream) is plotted as a function of location $x$ for several

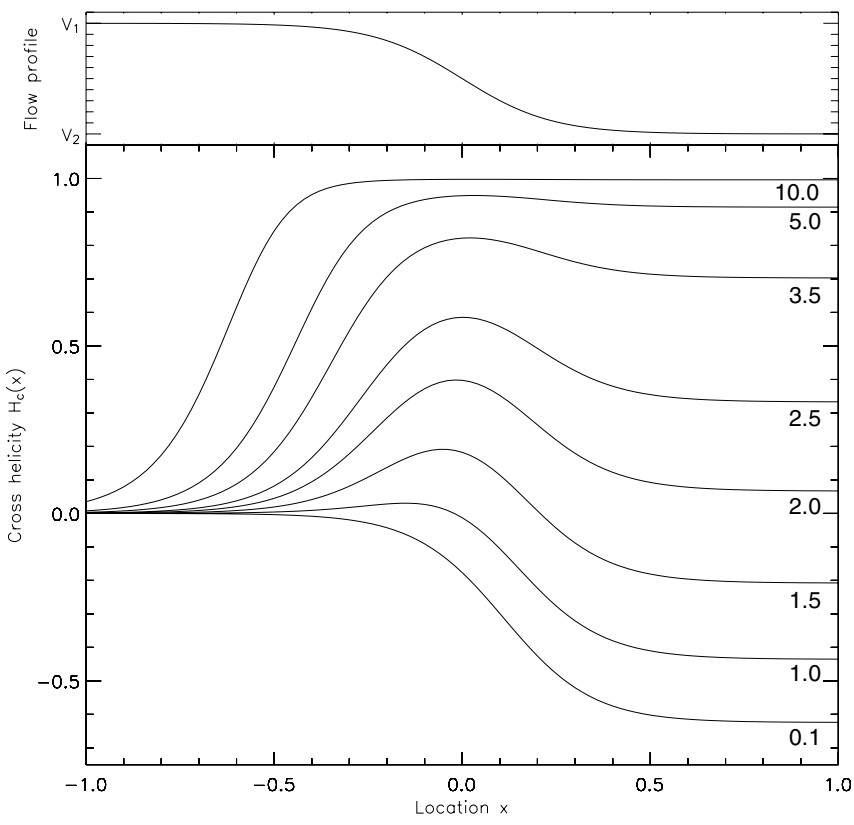

Fig. 2. Form of the flow profile (upper panel) and the local cross helicity across the shock when $M / M_{\mathrm{c}}=2$ (lower panel). Shock proper speeds $u_{1}$ range from $0.1 c$ to $10 c$ (lines from bottom to top, respectively). In the upper panel the flow speed is in arbitrary units, showing only the form of the transition from far upstream speed $V_{1}$ to that of the downstream, $V_{2}$, and the spatial range across which the transition takes place for both the flow speed and the cross-helicities. See text for details.

shocks with speeds ranging from non- to fully relativistic, but all having the Alfvénic Mach number $M=2 M_{\mathrm{c}}$. The critical Mach number $M_{\mathrm{c}}=\sqrt{r}$ is calculated separately for each shock speed following the scheme described in VVS03. The spectral index of the turbulence is $q=1.5$ for all cases.

\subsection{Decreased compressio ratio}

While the assumption of small-amplitude waves leaves the compression of the plasma itself unaffected, the compression felt by the scattering particles, on the other hand, can be changed remarkably. This is due to the fact that the particles are scattered by the turbulent waves, and if the waves have speed non-neglibigle compared to the speed of the flow (i.e. waves are not assumed to be frozen-in to the plasma), the speed profile of the scattering centres can be different from that of the plasma. Thus, also the effective compression ratio felt by the particles, $r_{k}$, can differ from the gas compression ratio $r$. Using the local cross-helicity $H_{\mathrm{c}}$ we can express the average wave speed as

$V_{k}(x)=\frac{V(x)+H_{\mathrm{c}}(x) V_{\mathrm{A}}(x)}{1+H_{\mathrm{c}}(x) V(x) V_{\mathrm{A}}(x)}$.

Now the scattering-centre compression ratio is calculated using the average wave speeds $\left(V_{k, 1}\right.$ and $\left.V_{k, 2}\right): r_{k}=V_{k, 1} / V_{k, 2}$.

As noted earlier, if the cross-helicity in the upstream equals \pm 1 it remains unchanged throughout the shock. So if there are e.g. only forward waves in the upstream, the upstream scattering centre speed is simply the (relativistic) sum of the flow speed and the local Alfvén speed; and likewise in the downstream. However, because of the compression and drop in the flow speed at the shock, the ratio of the Alfvén and the flow speed in the downstream is larger than in the upstream. Also its effect on the 


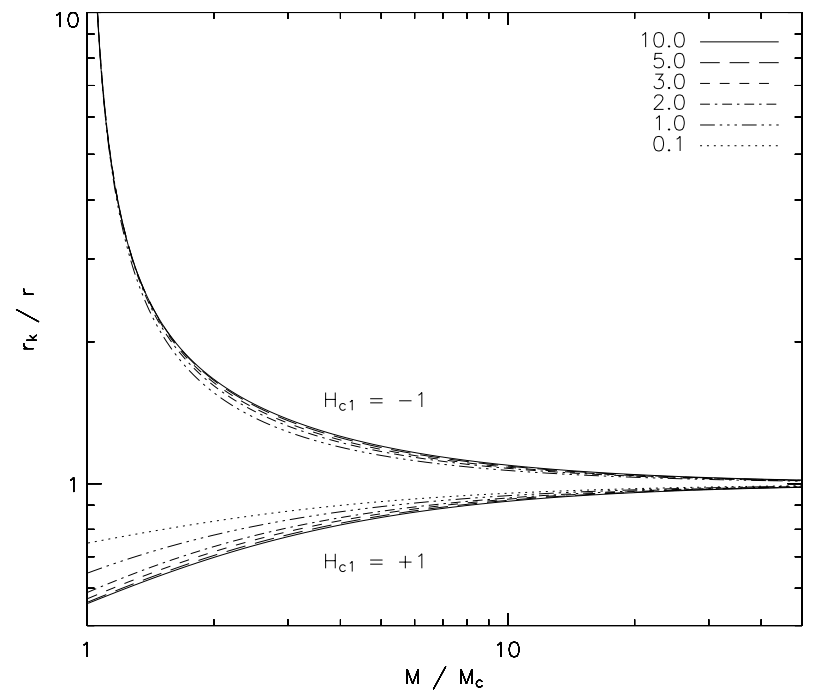

Fig. 3. Ratio of the scattering centre compression ratio $r_{k}$ to that of the gas $r$ for different shock proper speeds $u_{1}$ as functions of the Alvénic Mach number $M$. Lines are drawn for upstream cross-helicity $H_{\mathrm{c} 1}=-1$ (group of the uppermost lines) and +1 (lower group). The spectral index of the turbulence power spectrum is $q=1.5$ and $M_{\mathrm{c}}=\sqrt{r}$; see text for details.

resulting shock-frame scattering-centre speed is larger when the underlying flow speed is not close to the speed of light. For only forward waves in the upstream this results in $r_{k}<r$, and for only backward waves in the upstream $r_{k}>r$. When the Alfvén speed drops and becomes negligible compared to local flow speeds (i.e. $M \rightarrow \infty), r_{k} \rightarrow r$. This is illustrated in Fig. 3 .

For vanishing upstream cross-helicity, the behaviour in a nonrelativistic shock was examined by VV05 and it was shown to be very similar to that in step shocks. Now for thick relativistic shock, because of the positive downstream cross-helicity, the average shock-frame wave speed in the downstream is higher than the local flow speed, and, thus, the compression ratio of the scattering centres is lower than that of the gas.

Resulting scattering-centre compression ratios (scaled to the gas compression ratio) are plotted for upstream cross-helicities $H_{\mathrm{c}, 1}= \pm 1$ in Fig. 3, and for $H_{\mathrm{c}, 1}=0$ in Fig. 4. The nonrelativistic $\left(u_{1}=0.1\right)$ case is the same as in VV05 and, as for step shocks, the compression ratio increases also for higher speeds. However, on the contrary to what VV05 expected, for the fastest low- $M$ shocks the scattering-centre compression ratio does not increase steadily as $M \rightarrow M_{\mathrm{c}}$, but falls below the gas compression ratio when the shock speed becomes relativistic.

\subsection{Limitations for the analysis}

In earlier studies by Vainio \& Schlickeiser (1998) for nonrelativistic shocks and VVS03 for relativistic ones, the transmission properties were solved for waves of wavelengths essentially longer than the length scales of the shock. Here we have studied the opposite of "short" waves flowing through a shock thicker than the wave length scales. Although these two transmission schemes work differently and in different parts of parameter space, it would be physically meaningful to apply both schemes in the same shock: the analysis of VVS03 for the part of the turbulence spectrum for which the wavelengths are longer than the width of the shock transition, and the method described in this paper for waves for which the plasma parameters vary slowly

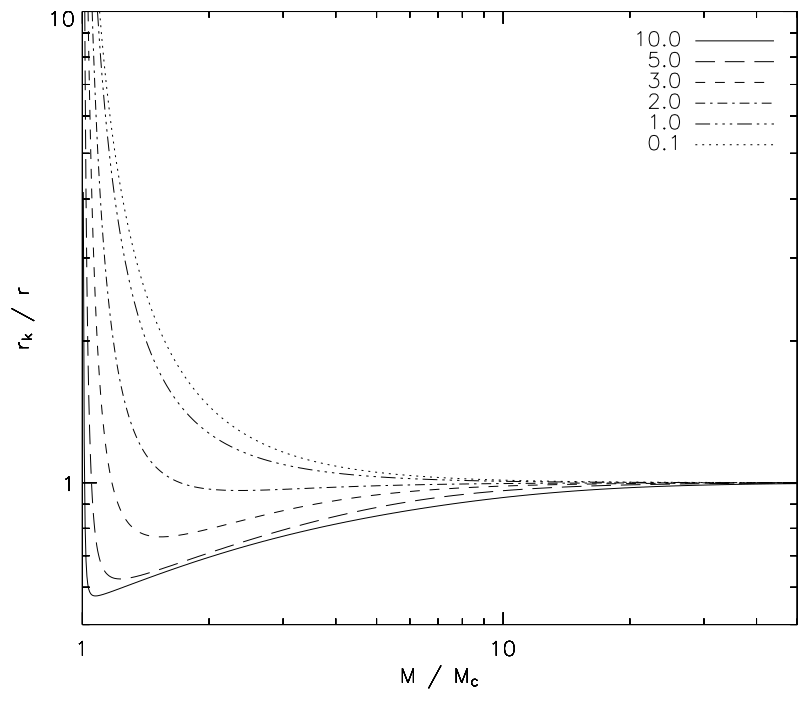

Fig. 4. Same as Fig. 3, but for $H_{\mathrm{c} 1}=-0$.
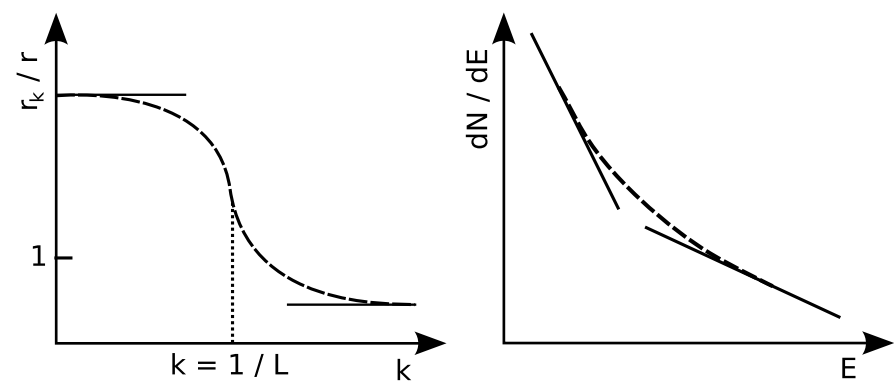

Fig. 5. Sketch of the scattering-centre compression ratio as a function of wavenumber (left) and the resulting accelerated particle spectrum (right) in a thick relativistic shock. $L$ denotes the shock thickness.

throughout the transition. Thus, the scattering-centre compression ratio is a function of wavenumber, and the resulting accelerated particle spectrum is not a simple power-law in energy (Fig. 5).

As the study here assumed no back-reaction of the waves to the shock structure or dynamics, the approach is limited to small-amplitude waves. The test-wave approach, on the other hand, leads to mathematical singularities at $M \rightarrow M_{\mathrm{c}}=\sqrt{r}$; at this limit the antiparallel waves are amplified infinitely. In the analysis this limitation affects through the simplified calculation of the gas compression ratio. In more detailed further analysis the effect of the upstream wave parameters to the calculation of the gas compression ratio should be taken into account in order to remove the (non-physical) singularities, as demonstrated by Vainio \& Schlickeiser (1999) for nonrelativistic step shocks.

Also the inclusion of accelerating particles will have effects on the waves; in this treatise the effect of the particles on the waves have been omitted. However, the resonant wave-particle interactions could have a damping effect on the turbulence, thus rapidly changing the transmitted wave distributions, especially when moving downstream away from the shock. Similarily, our analysis also neglects the wave-wave interactions in the downstream medium (Vainio \& Spanier 2005).

Finally we merely note that in the present study we have confined the analysis to strictly parallel shocks. For oblique magnetic field alignments the treatment of the turbulence 
transmission becomes very complicated and is beyond the scope of this study.

\section{Conclusions}

We have calculated the transmission coefficients for Alfvén (test) waves with short wavelengths through a modified shock with background plasma properties changing slowly compared to the wave length scales.

While the transmission trough a step shock was found similar throughout the whole shock speed range (VVS03), and, additionally, very similar behaviour was observed also for thick shocks in the nonrelativistic limit (VV05), qualitative and signifigant differences emerge for thick shocks when the speed increases. As a consequence, from the point of view of the particle acceleration studies, the compression felt by the particles (i.e. that of the scattering centres) at a thick relativistic low- $M$ shock was shown to be weaker than the compression of the background flow. For slower (from non- to mildly relativistic) shocks the scattering centre compression ratio was shown to increase, like in step shocks.
Acknowledgements. The authors thank an anonymous referee for valuable suggestions on how to improve the manuscript. J.T. further thanks professors Reinhard Schlickeiser and Ian Lerche for constructive criticism and inspiring discussions.

\section{References}

Achterberg, A. 1983, Phys. Rev. A, 28, 2449

Bell, A. R. 1978, MNRAS, 182, 147

Dewar, R. L. 1970, Phys. Fluids, 13, 2710

Drury, L. O’C. 1983, Rep. Prog. Phys., 46, 973

Drury, L. O’C., Axford W. I., \& Summers D. 1982, MNRAS 198, 833

Keshet, U., \& Waxman, E. 2005, Phys. Rev. Lett., 94, 111102

Kirk, J. G., \& Duffy, P. 1999, J. Phys. G, 25, R163

Schneider, P., \& Kirk, J. G. 1989, A\&A, 217, 344

Vainio, R., \& Schlickeiser, R. 1998, A\&A, 331, 793

Vainio, R., \& Schlickeiser, R. 1999, A\&A, 343, 303

Vainio, R. \& Spanier, F. 2005, A\&A, 437, 1

Vainio, R., Virtanen, J. J. P., \& Schlickeiser, R. 2003 (VVS03), A\&A, 409, 821

Vainio, R., Virtanen, J. J. P., \& Schlickeiser, R. 2005, A\&A, 431, 7

Virtanen, J. J. P., \& Vainio, R. 2005a, A\&A, 439, 461 (VV05)

Virtanen, J. J. P., \& Vainio, R. 2005b, ApJ, 621, 313 